PROCEEDINGS OF THE

AMERICAN MATHEMATICAL SOCIETY

Volume 135, Number 11, November 2007, Pages 3571-3579

S 0002-9939(07)08882-X

Article electronically published on June 21, 2007

\title{
MATRIX SUMMABILITY AND UNIFORM CONVERGENCE OF SERIES
}

\author{
ANTONIO AIZPURU, FRANCISCO J. GARCÍA-PACHECO, \\ AND CONSUELO PÉREZ-ESLAVA
}

(Communicated by N. Tomczak-Jaegermann)

\begin{abstract}
Some classical results about uniform convergence of unconditionally convergent series are generalized to weakly unconditionally Cauchy series by means of the matrix summability method for regular matrices.
\end{abstract}

\section{INTRODUCTION AND BACKGROUND}

A series $\sum_{k=1}^{\infty} x_{k}$ in a real Banach space $X$ is called weakly unconditionally Cauchy ( $\omega \mathrm{uC}$ ) if $\sum_{k=1}^{\infty}\left|f\left(x_{k}\right)\right|<\infty$ for every $f \in X^{*}$, and is called unconditionally convergent (uc) if $\sum_{k=1}^{\infty} x_{\pi(k)}$ is convergent for every permutation $\pi$ of $\mathbb{N}$. It is well known (see [7] and [8]) that a series $\sum_{k=1}^{\infty} x_{k}$ is uc if and only if the series $\sum_{k=1}^{\infty} a_{k} x_{k}$ is convergent for every $\left(a_{k}\right)_{k \in \mathbb{N}} \in \ell_{\infty}$, and is $\omega \mathrm{uC}$ if and only if the series $\sum_{k=1}^{\infty} a_{k} x_{k}$ is convergent for every $\left(a_{k}\right)_{k \in \mathbb{N}} \in c_{0}$. It is also well known (see [4] and [7]) that a Banach space has a copy of $c_{0}$ if and only if it possesses a $\omega \mathrm{uC}$ series which is not uc. Another characterization of $\omega \mathrm{uC}$ series that appears in 7] states that a series $\sum_{k=1}^{\infty} x_{k}$ is $\omega \mathrm{uC}$ if and only if

$$
\sup \left\{\left\|\sum_{k=1}^{n} a_{k} x_{i}\right\|:\left|a_{k}\right| \leq 1, k \in\{1, \ldots, n\}, n \in \mathbb{N}\right\}<\infty .
$$

Now, let $X$ denote a real Banach space. Given a regular matrix $A=\left(\alpha_{i j}\right)_{i, j \in \mathbb{N}}$ and a vector subspace $\mathcal{S}$ of $\ell_{\infty}$ containing $c_{0}$, the following space of vector sequences is defined:

$$
X(\mathcal{S}, A)=\left\{\left(x_{k}\right)_{k \in \mathbb{N}} \text { in } X: A \sum_{k=1}^{\infty} a_{k} x_{k} \text { exists for every }\left(a_{k}\right)_{k \in \mathbb{N}} \in \mathcal{S}\right\},
$$

where

$$
A \sum_{k=1}^{\infty} a_{k} x_{k}=\lim _{i \rightarrow \infty} \sum_{j=1}^{\infty} \alpha_{i j}\left(\sum_{k=1}^{j} a_{k} x_{k}\right) .
$$

We refer the reader to [5] for a wider perspective on matrix summability methods. Following the notation in [1], we let $X\left(c_{0}\right)$ denote the space of weakly unconditionally Cauchy series in $X$, also denoted by $\ell_{1}^{\omega}(X)$, and $X\left(\ell_{\infty}\right)$ will be the space

Received by the editors January 17, 2006 and, in revised form, August 9, 2006.

2000 Mathematics Subject Classification. Primary 46B15, 46B25, 46B20.

Key words and phrases. Uniform convergence, unconditionally convergent series, weakly unconditionally Cauchy series, matrix summability, regular matrices.

(C)2007 American Mathematical Society 3571 
of unconditionally convergent series in $X$. Both spaces are real Banach spaces endowed with the norm

$$
\left\|\left(x_{k}\right)_{k \in \mathbb{N}}\right\|=\sup \left\{\left\|\sum_{k=1}^{n} a_{k} x_{k}\right\|:\left|a_{k}\right| \leq 1, k \in\{1, \ldots, n\}, n \in \mathbb{N}\right\} .
$$

Furthermore, $X\left(\ell_{\infty}\right) \subseteq X(\mathcal{S}, A) \subseteq X\left(c_{0}\right)$. Also, notice that if $A$ is the identity matrix, then $X(\mathcal{S}, A)$ is exactly the space $X(\mathcal{S})$ studied in 1 .

On the other hand, in 11 is shown the following representative result concerning uniform convergence of unconditionally convergent series.

Theorem 1.1. Let $X$ be a real Banach space. Let $\left(x^{n}\right)_{n \in \mathbb{N}}$ be a sequence in $X\left(\ell_{\infty}\right)$ such that for every $(a(k))_{k \in \mathbb{N}} \in \ell_{\infty}, \lim _{n \rightarrow \infty} \sum_{k=1}^{\infty} a(k) x^{n}(k)$ exists. Then, there exists $x^{0} \in X\left(\ell_{\infty}\right)$ such that $\lim _{n \rightarrow \infty}\left\|x^{n}-x^{0}\right\|=0$ in $X\left(\ell_{\infty}\right)$.

In [1] and 2] several similar results are proved for the case of weakly unconditionally Cauchy series. In this manuscript, we mean to show some other results on uniform convergence of weakly unconditionally Cauchy series.

\section{Preliminary Results}

The first result we would like to present shows the completeness of the space $X(\mathcal{S}, A)$ endowed with the norm given in (1.1).

Theorem 2.1. Let $X$ be a real Banach space and consider $A=\left(\alpha_{i j}\right)_{i, j \in \mathbb{N}}$ to be a regular matrix and $\mathcal{S}$ a vector subspace of $\ell_{\infty}$ containing $c_{0}$. Then, $X(\mathcal{S}, A)$ is a complete space endowed with the norm given in (1.1).

Proof. We will show that $X(\mathcal{S}, A)$ is closed in $X\left(c_{0}\right)$. Let us start by denoting

$$
M=\sup \left\{\sum_{j=1}^{\infty}\left|\alpha_{i j}\right|: i \in \mathbb{N}\right\} \text {. }
$$

Note that $0<M<\infty$ since $A$ is regular. Now, take $\left(x^{n}\right)_{n \in \mathbb{N}}$ to be a sequence in $X(\mathcal{S}, A)$ and $x^{0} \in X\left(c_{0}\right)$ such that $\left\|x^{n}-x^{0}\right\| \rightarrow 0$ as $n \rightarrow \infty$ in $X\left(c_{0}\right)$. We will see that $x^{0} \in X(\mathcal{S}, A)$. Let us fix an arbitrary element $a=(a(k))_{k \in \mathbb{N}} \in \mathcal{S}$. For every $n \in \mathbb{N}$, there exists $x_{n} \in X$ such that

$$
\lim _{i \rightarrow \infty} \sum_{j=1}^{\infty} \alpha_{i j}\left(\sum_{k=1}^{j} a(k) x^{n}(k)\right)=x_{n} .
$$

Let us show that $\left(x_{n}\right)_{n \in \mathbb{N}}$ is a Cauchy sequence in $X$. Let $\varepsilon>0$. Since $\left(x^{n}\right)_{n \in \mathbb{N}}$ is a Cauchy sequence in $X\left(c_{0}\right)$, we can find $n_{0} \in \mathbb{N}$ so that $\left\|x^{p}-x^{q}\right\| \leq \varepsilon /(M(\|a\|+1))$ if $p, q \geq n_{0}$. Then, for arbitrarily fixed $p, q \geq n_{0}$, we have

$$
\begin{aligned}
\left\|\sum_{j=1}^{\infty} \alpha_{i j}\left(\sum_{k=1}^{j} a(k)\left(x^{p}(k)-x^{q}(k)\right)\right)\right\| & \leq \sum_{j=1}^{\infty}\left|\alpha_{i j}\right|\left\|\sum_{k=1}^{j} a(k)\left(x^{p}(k)-x^{q}(k)\right)\right\| \\
& \leq M\|a\|\left\|x^{p}-x^{q}\right\| \\
& \leq \varepsilon
\end{aligned}
$$


for every $i \in \mathbb{N}$. Now, by taking into account that

$$
\left\|x_{p}-x_{q}\right\|=\lim _{i \rightarrow \infty}\left\|\sum_{j=1}^{\infty} \alpha_{i j}\left(\sum_{k=1}^{j} a(k)\left(x^{p}(k)-x^{q}(k)\right)\right)\right\|,
$$

we deduce that $\left\|x_{p}-x_{q}\right\| \leq \varepsilon$ for $p, q \geq n_{0}$, and hence $\left(x_{n}\right)_{n \in \mathbb{N}}$ is a Cauchy sequence in $X$. Since $X$ is complete, $\left(x_{n}\right)_{n \in \mathbb{N}}$ is convergent to some $x_{0} \in X$. Finally, we will show that

$$
\lim _{i \rightarrow \infty} \sum_{j=1}^{\infty} \alpha_{i j}\left(\sum_{k=1}^{j} a(k) x^{0}(k)\right)=x_{0},
$$

and we will be done. Let $\varepsilon>0$. Let us fix $p \in \mathbb{N}$ with $\left\|x^{p}-x^{0}\right\| \leq \varepsilon /(3 M(\|a\|+1))$ and $\left\|x_{p}-x_{0}\right\| \leq \frac{\varepsilon}{3}$. Now, there exists $i_{0} \in \mathbb{N}$ verifying that

$$
\left\|\sum_{j=1}^{\infty} \alpha_{i j}\left(\sum_{k=1}^{j} a(k) x^{p}(k)\right)-x_{p}\right\| \leq \frac{\varepsilon}{3}
$$

for every $i \geq i_{0}$. Therefore,

$$
\begin{aligned}
\left\|\sum_{j=1}^{\infty} \alpha_{i j}\left(\sum_{k=1}^{j} a(k) x^{0}(k)\right)-x_{0}\right\| & \leq \sum_{j=1}^{\infty}\left|\alpha_{i j}\right|\left\|\sum_{k=1}^{j} a(k)\left(x^{0}(k)-x^{p}(k)\right)\right\| \\
& +\left\|\sum_{j=1}^{\infty} \alpha_{i j}\left(\sum_{k=1}^{j} a(k) x^{p}(k)\right)-x_{p}\right\| \\
& +\left\|x_{p}-x_{0}\right\| \\
& \leq \frac{\varepsilon}{3}+\frac{\varepsilon}{3}+\frac{\varepsilon}{3} \\
& =\frac{\varepsilon}{}
\end{aligned}
$$

for every $i \geq i_{0}$. As a consequence, the result is proved.

Now, we will introduce another space of vector sequences. Let $X$ denote a real Banach space. Given a regular matrix $A=\left(\alpha_{i j}\right)_{i, j \in \mathbb{N}}$ and a vector subspace $\mathcal{S}$ of $\ell_{\infty}$ containing $c_{0}$, the following space of vector sequences is defined:

$$
X_{\omega}(\mathcal{S}, A)=\left\{\left(x_{k}\right)_{k \in \mathbb{N}} \text { in } X: \omega A \sum_{k=1}^{\infty} a_{k} x_{k} \text { exists for every }\left(a_{k}\right)_{k \in \mathbb{N}} \in \mathcal{S}\right\},
$$

where

$$
\omega A \sum_{k=1}^{\infty} a_{k} x_{k}=\omega \lim _{i \rightarrow \infty} \sum_{j=1}^{\infty} \alpha_{i j}\left(\sum_{k=1}^{j} a_{k} x_{k}\right) .
$$

In [3] it is proved that a series $\sum_{k=1}^{\infty} x_{k}$ is $\omega \mathrm{uC}$ if and only if $\omega A \sum_{k=1}^{\infty} a_{k} x_{k}$ exists for every $\left(a_{k}\right)_{k \in \mathbb{N}} \in c_{0}$. Notice that $X\left(\ell_{\infty}\right) \subseteq X(\mathcal{S}, A) \subseteq X_{\omega}(\mathcal{S}, A) \subseteq X\left(c_{0}\right)$. In the next theorem, we show that the space $X_{\omega}(\mathcal{S}, A)$ is complete endowed with the norm given in (1.1).

Theorem 2.2. Let $X$ be a real Banach space and consider $A=\left(\alpha_{i j}\right)_{i, j \in \mathbb{N}}$ to be a regular matrix and $\mathcal{S}$ a vector subspace of $\ell_{\infty}$ containing $c_{0}$. Then, $X_{\omega}(\mathcal{S}, A)$ is a complete space endowed with the norm given in (1.1). 
Proof. As before, we will show that $X_{\omega}(\mathcal{S}, A)$ is closed in $X\left(c_{0}\right)$. Again

$$
M=\sup \left\{\sum_{j=1}^{\infty}\left|\alpha_{i j}\right|: i \in \mathbb{N}\right\} .
$$

Take $\left(x^{n}\right)_{n \in \mathbb{N}}$ to be a sequence in $X_{\omega}(\mathcal{S}, A)$ and $x^{0} \in X\left(c_{0}\right)$ such that $\left\|x^{n}-x^{0}\right\| \rightarrow$ 0 as $n \rightarrow \infty$ in $X\left(c_{0}\right)$. We will see that $x^{0} \in X_{\omega}(\mathcal{S}, A)$. Let us fix an arbitrary element $a=(a(k))_{k \in \mathbb{N}} \in \mathcal{S}$. For every $n \in \mathbb{N}$, there exists $x_{n} \in X$ such that

$$
\omega \lim _{i \rightarrow \infty} \sum_{j=1}^{\infty} \alpha_{i j}\left(\sum_{k=1}^{j} a(k) x^{n}(k)\right)=x_{n} .
$$

Let us see that $\left(x_{n}\right)_{n \in \mathbb{N}}$ is a Cauchy sequence in $X$. Let $\varepsilon>0$. Since $\left(x^{n}\right)_{n \in \mathbb{N}}$ is a Cauchy sequence in $X\left(c_{0}\right)$, we can find $n_{0} \in \mathbb{N}$ so that $\left\|x^{p}-x^{q}\right\| \leq \varepsilon /(M(\|a\|+1))$ if $p, q \geq n_{0}$. Also, there is $g \in \mathrm{S}_{X^{*}}$ with $\left\|x_{p}-x_{q}\right\|=\left|g\left(x_{p}-x_{q}\right)\right|$. Then, for arbitrarily fixed $p, q \geq n_{0}$, we have

$$
\begin{aligned}
\mid \sum_{j=1}^{\infty} & \alpha_{i j}\left(\sum_{k=1}^{j} a(k) g\left(\left(x^{p}(k)-x^{q}(k)\right)\right)\right) \mid \\
\leq & \left\|\sum_{j=1}^{\infty} \alpha_{i j}\left(\sum_{k=1}^{j} a(k)\left(x^{p}(k)-x^{q}(k)\right)\right)\right\| \\
& \leq \sum_{j=1}^{\infty}\left|\alpha_{i j}\right|\left\|\sum_{k=1}^{j} a(k)\left(x^{p}(k)-x^{q}(k)\right)\right\| \\
& \leq M\|a\|\left\|x^{p}-x^{q}\right\| \\
& \leq \varepsilon
\end{aligned}
$$

for every $i \in \mathbb{N}$. Now, by taking into account that

$$
\begin{aligned}
\left\|x_{p}-x_{q}\right\| & =\left|g\left(x_{p}-x_{q}\right)\right| \\
& =\left|\lim _{i \rightarrow \infty} \sum_{j=1}^{\infty} \alpha_{i j}\left(\sum_{k=1}^{j} a(k) g\left(\left(x^{p}(k)-x^{q}(k)\right)\right)\right)\right|,
\end{aligned}
$$

we deduce that $\left\|x_{p}-x_{q}\right\| \leq \varepsilon$ for $p, q \geq n_{0}$, and hence $\left(x_{n}\right)_{n \in \mathbb{N}}$ is a Cauchy sequence in $X$. Since $X$ is complete, $\left(x_{n}\right)_{n \in \mathbb{N}}$ is convergent to some $x_{0} \in X$. Finally, we will show that

$$
\omega \lim _{i \rightarrow \infty} \sum_{j=1}^{\infty} \alpha_{i j}\left(\sum_{k=1}^{j} a(k) x^{0}(k)\right)=x_{0},
$$

and we will be done. Let $\varepsilon>0$ and $f \in \mathrm{S}_{X^{*}}$. Let us fix $p \in \mathbb{N}$ with $\left\|x^{p}-x^{0}\right\| \leq$ $\varepsilon /(3 M(\|a\|+1))$ and $\left\|x_{p}-x_{0}\right\| \leq \frac{\varepsilon}{3}$. Now, there exists $i_{0} \in \mathbb{N}$ verifying that

$$
\left\|\sum_{j=1}^{\infty} \alpha_{i j}\left(\sum_{k=1}^{j} a(k) f\left(x^{p}(k)\right)\right)-f\left(x_{p}\right)\right\| \leq \frac{\varepsilon}{3},
$$


for every $i \geq i_{0}$. Therefore,

$$
\begin{aligned}
\| \sum_{j=1}^{\infty} \alpha_{i j} & \left(\sum_{k=1}^{j} a(k) f\left(x^{0}\right)(k)\right)-f\left(x_{0}\right) \| \\
\leq & \sum_{j=1}^{\infty}\left|\alpha_{i j}\right|\left\|\sum_{k=1}^{j} a(k)\left(x^{0}(k)-x^{p}(k)\right)\right\| \\
& +\left\|\sum_{j=1}^{\infty} \alpha_{i j}\left(\sum_{k=1}^{j} a(k) f\left(x^{p}\right)(k)\right)-f\left(x_{p}\right)\right\| \\
& +\left\|f\left(x_{p}\right)-f\left(x_{0}\right)\right\| \\
\leq & \frac{\varepsilon}{3}+\frac{\varepsilon}{3}+\frac{\varepsilon}{3} \\
= & \varepsilon,
\end{aligned}
$$

for every $i \geq i_{0}$. As a consequence, the result is proved.

\section{MAin Results}

In this section, we will show two sufficient conditions for the uniform convergence of weakly unconditionally Cauchy sequences. Both results could be considered as general versions of Theorem 1.1 for weakly unconditionally Cauchy series.

Lemma 3.1. Let $X$ be a real Banach space and consider $A=\left(\alpha_{i j}\right)_{i, j \in \mathbb{N}}$ to be a regular matrix and $\mathcal{S}$ a vector subspace of $\ell_{\infty}$ containing $c_{0}$. For every vector sequence $x=(x(k))_{k \in \mathbb{N}} \in X_{\omega}(\mathcal{S}, A)$, the linear mapping

$$
\begin{gathered}
\sigma_{x}: \mathcal{S} \longrightarrow X \\
a=(a(k))_{k \in \mathbb{N}} \longmapsto \sigma_{x}(a)=A \sum_{k=1}^{\infty} a(k) x(k)
\end{gathered}
$$

is continuous with $\left\|\sigma_{x}\right\| \leq M\|x\|$.

Proof. Let us consider

$$
M=\sup \left\{\sum_{j=1}^{\infty}\left|\alpha_{i j}\right|: i \in \mathbb{N}\right\} .
$$

Let $a=(a(k))_{k \in \mathbb{N}} \in \mathcal{S}$. There exists $f \in \mathrm{S}_{X^{*}}$ satisfying

$$
\begin{aligned}
\left\|\sigma_{x}(a)\right\| & =\left|f\left(\sigma_{x}(a)\right)\right| \\
& =\left|\lim _{i \rightarrow \infty} \sum_{j=1}^{\infty} \alpha_{i j}\left(\sum_{k=1}^{j} a(k) f(x(k))\right)\right| .
\end{aligned}
$$

Now, for every $i \in \mathbb{N}$, we have

$$
\begin{aligned}
\left|\sum_{j=1}^{\infty} \alpha_{i j}\left(\sum_{k=1}^{j} a(k) f(x(k))\right)\right| & \leq\left\|\sum_{j=1}^{\infty} \alpha_{i j}\left(\sum_{k=1}^{j} a(k) x(k)\right)\right\| \\
& \leq M\|a\|\|x\| .
\end{aligned}
$$


As a consequence, $\left\|\sigma_{x}(a)\right\| \leq M\|a\|\|x\|$, which means that $\sigma_{x}$ is continuous with $\left\|\sigma_{x}\right\| \leq M\|x\|$.

Remark 3.2. Before stating the first main theorem, let us make some remarks related to regular matrices and uniform convergence.

(1) Keeping the same notation as in the previous lemma, for every vector sequence $x=(x(k))_{k \in \mathbb{N}} \in X(\mathcal{S}, A)$, the linear mapping

$$
\begin{gathered}
\sigma_{x}: \mathcal{S} \longrightarrow X \\
a=(a(k))_{k \in \mathbb{N}} \longmapsto \sigma_{x}(a)=A \sum_{k=1}^{\infty} a(k) x(k)
\end{gathered}
$$

is continuous with $\left\|\sigma_{x}\right\| \leq M\|x\|$.

(2) It is well known that if $\sum_{k=1}^{\infty} x_{k}=x_{0}$ and $A$ is a regular matrix, then $A \sum_{k=1}^{\infty} x_{k}=x_{0}$. However, the converse is not true in general. It is also possible that $\omega A \sum_{k=1}^{\infty} x_{k}$ exists but not $\omega \sum_{k=1}^{\infty} x_{k}$. Therefore, it is possible that $\left(x_{k}\right)_{k \in \mathbb{N}} \in X(\mathcal{S}, A)$, but for some $\left(a_{k}\right)_{k \in \mathbb{N}} \in \mathcal{S}, \sum_{k=1}^{\infty} a_{k} x_{k}$ does not exist. Similarly, it is possible that $\left(x_{k}\right)_{k \in \mathbb{N}} \in X_{\omega}(\mathcal{S}, A)$, but for some $\left(a_{k}\right)_{k \in \mathbb{N}} \in \mathcal{S}, \omega \sum_{k=1}^{\infty} a_{k} x_{k}$ does not exist either.

Next, we will present the first one of our main results in this paper, in which we deduce, from a pointwise convergence situation, the existence of uniform convergence. More specifically, we obtain a sufficient condition for the convergence of sequences in $X\left(c_{0}\right)$. Nevertheless, before stating this result, we need first to recall some concepts. We will say (see [2]) that a real Banach space $X$ is an $M$ Grothendieck space, where $M$ is a vector subspace of $X^{* *}$ containing $X$, if every $\sigma\left(X^{*}, X\right)$-convergent sequence in $X^{*}$ is $\sigma\left(X^{*}, M\right)$-convergent. When $M=X^{* *}$, we just say that $X$ is a Grothendieck space. Let us observe also that if $\mathcal{S}$ is a vector subspace of $\ell_{\infty}$ containing $c_{0}$, then $\ell_{\infty}$ can be identified with a vector subspace of $S^{* *}$. Indeed, we can identify any $\left(a_{k}\right)_{k \in \mathbb{N}} \in \ell_{\infty}$ with the mapping

$$
\begin{aligned}
\mathcal{S}^{*} & \longrightarrow \mathbb{R} \\
g & \longmapsto \sum_{k=1}^{\infty} a_{k} g\left(e^{k}\right),
\end{aligned}
$$

where $\left(e^{k}\right)_{k \in \mathbb{N}}$ denotes the canonical basis of $c_{0}$. Therefore, if $\mathcal{S}$ is a closed vector subspace of $\ell_{\infty}$ containing $c_{0}$, it does make sense to consider the possibility for $\mathcal{S}$ of being an $\ell_{\infty}$-Grothendieck space.

Theorem 3.3. Let $X$ be a real Banach space. Let $\left(x^{n}\right)_{n \in \mathbb{N}}$ be a sequence in $X\left(c_{0}\right)$ satisfying the following:

(1) There exists a closed vector subspace $\mathcal{S}$ of $\ell_{\infty}$ containing $c_{0}$ which is an $\ell_{\infty}$-Grothendieck space.

(2) There exists a regular matrix $A=\left(\alpha_{i j}\right)_{i, j \in \mathbb{N}}$ such that

$$
\lim _{n \rightarrow \infty} \omega A \sum_{k=1}^{\infty} a(k) x^{n}(k)
$$

exists for every $(a(k))_{k \in \mathbb{N}} \in \mathcal{S}$.

Then, there exists $x^{0} \in X\left(c_{0}\right)$ such that $\lim _{n \rightarrow \infty}\left\|x^{n}-x^{0}\right\|=0$ in $X\left(c_{0}\right)$. 
Proof. Since $X\left(c_{0}\right)$ is complete, it suffices to show that $\left(x^{n}\right)_{n \in \mathbb{N}}$ is a Cauchy sequence in $X\left(c_{0}\right)$. On the contrary, let us assume that it is not. There exists $\varepsilon>0$ and an increasing sequence $\left(n_{m}\right)_{m \in \mathbb{N}}$ of naturals such that if $z^{m}:=x^{n_{m}}-x^{n_{m+1}}$, then $\left\|z_{m}\right\|>\varepsilon$. Now, for every $m \in \mathbb{N}$ there exists $f_{m} \in \mathrm{S}_{X^{*}}$ such that

$$
\sum_{k=1}^{\infty}\left|f_{m}\left(z^{m}(k)\right)\right|>\varepsilon
$$

Again for every $m \in \mathbb{N}$, we consider the continuous linear mapping $\sigma_{z^{m}}$ defined in (3.1), which will be simply denoted as $\sigma_{m}$. Let us observe that, for every $a=$ $(a(k))_{k \in \mathbb{N}} \in \mathcal{S}$, we have

$$
\begin{aligned}
\lim _{m \rightarrow \infty} \sigma_{m}(a)= & \lim _{m \rightarrow \infty}\left(\omega A \sum_{k=1}^{\infty} a(k) x^{n_{m}}(k)\right) \\
& -\lim _{m \rightarrow \infty}\left(\omega A \sum_{k=1}^{\infty} a(k) x^{n_{m+1}}(k)\right) \\
= & 0 ;
\end{aligned}
$$

therefore $\left(f_{m} \circ \sigma_{m}\right)_{m \in \mathbb{N}}$ is a sequence in $\mathcal{S}^{*}$ which is $\omega^{*}$-convergent to 0 . By hypothesis, $\left(f_{m} \circ \sigma_{m}\right)_{m \in \mathbb{N}}$ is $\left(\mathcal{S}^{*}, \ell_{\infty}\right)$-convergent to 0 . According to mapping (3.2), this means that if $(a(k))_{k \in \mathbb{N}} \in \ell_{\infty}$, then

$$
\lim _{m \rightarrow \infty} \sum_{k=1}^{\infty} a(k)\left(f_{m} \circ \sigma_{m}\right)\left(e^{k}\right)=0 .
$$

Now, for every $m, k \in \mathbb{N}$, we have that

$$
\begin{aligned}
\left(f_{m} \circ \sigma_{m}\right)\left(e^{k}\right) & =f_{m}\left(\omega A \sum_{l=1}^{\infty} e^{k}(l) z^{m}(l)\right) \\
& =f_{m}\left(\omega \lim _{i \rightarrow \infty} \sum_{j=1}^{\infty} \alpha_{i j}\left(\sum_{l=1}^{j} e^{k}(l) z^{m}(l)\right)\right) \\
& =f_{m}\left(\omega \lim _{i \rightarrow \infty}\left(\sum_{j=k}^{\infty} \alpha_{i j}\right) z^{m}(k)\right) \\
& =\lim _{i \rightarrow \infty}\left(\sum_{j=k}^{\infty} \alpha_{i j}\right) f_{m}\left(z^{m}(k)\right) \\
& =\lim _{i \rightarrow \infty}\left(\sum_{j=1}^{\infty} \alpha_{i j}-\sum_{j=1}^{k-1} \alpha_{i j}\right) f_{m}\left(z^{m}(k)\right) \\
& =f_{m}\left(z^{m}(k)\right)(\text { since } A \text { is regular }) .
\end{aligned}
$$

Next, note that $\left(\left(f_{m}\left(z^{m}(k)\right)_{k \in \mathbb{N}}\right)_{m \in \mathbb{N}}\right)$ is a sequence in $\ell_{1}$ verifying that, for every $(a(k))_{k \in \mathbb{N}} \in \ell_{\infty}$

$$
\lim _{m \rightarrow \infty} \sum_{k=1}^{\infty} a(k) f_{m}\left(z^{m}(k)\right)=0 ;
$$


that is, it is $\omega$-convergent to 0 . Schur's theorem then allows us to deduce that the sequence $\left(\left(f_{m}\left(z^{m}(k)\right)_{k \in \mathbb{N}}\right)_{m \in \mathbb{N}}\right)$ is convergent to 0 in $\ell_{1}$, but this fact contradicts (3.3).

Remark 3.4. Before stating the other main result of this paper, let us make some remarks about the previous theorem and Boolean algebras.

(1) If $A$ is the identity matrix, then the previous theorem generalizes Theorem 1.1 of Swartz and other results in [1] and [6].

(2) Let us consider $\mathcal{F}$ to be a Boolean subalgebra of $\mathcal{P}(\mathbb{N})$ verifying that $\psi_{0}(\mathbb{N}):=\{A \subseteq \mathbb{N}: \operatorname{card}(A)<\infty\} \subseteq \mathcal{F}$ (in [2] such Boolean subalgebras are called natural Boolean algebras). Let $T$ be the Stone space of $\mathcal{F}$. It is known (see [10]) that the corresponding space of real-valued continuous functions on $T, \mathcal{C}(T)$, can be identified with a subspace of $\ell_{\infty}$. According to [10], $\mathcal{F}$ is said to have the Grothendieck property if $\mathcal{C}(T)$ has the Grothendieck property; $\mathcal{F}$ is said to have the Nikodym property if the space $\mathcal{C}_{0}(T)$, of real-valued continuous functions on $T$ of finite range, is barrelled; and $\mathcal{F}$ is said to have the Vitali-Hahn-Saks property if it has both the Grothendieck and Nikodym properties.

(3) There exists a closed vector subspace $\mathcal{S}$ of $\ell_{\infty}$ containing $c_{0}$ which does not have a copy of $\ell_{\infty}$ and possesses the Vitali-Hahn-Saks property. This space was due to Hayden (see [9,) who obtained, by using transfinite induction, a particular subalgebra $\mathcal{F}$ of $\mathcal{P}(\mathbb{N})$ with $\psi_{0}(\mathbb{N}) \subseteq \mathcal{F}$.

Finally, it is time to present the second main result in this paper, in which we provide a sufficient condition for the convergence of sequences in $X\left(c_{0}\right)$ by means of pointwise convergence in natural Boolean algebras.

Theorem 3.5. Let $X$ be a real Banach space. Let $\left(x^{n}\right)_{n \in \mathbb{N}}$ be a sequence in $X\left(c_{0}\right)$. Consider a regular matrix $A=\left(\alpha_{i j}\right)_{i, j \in \mathbb{N}}$ and a natural Boolean algebra $\mathcal{F}$ with the Vitali-Hahn-Saks property. Assume that, for every $B \in \mathcal{F}$,

$$
\lim _{n \rightarrow \infty} \omega A \sum_{k \in B} x^{n}(k)
$$

exists. Then, there exists $x^{0} \in X\left(c_{0}\right)$ such that $\lim _{n \rightarrow \infty}\left\|x^{n}-x^{0}\right\|=0$ in $X\left(c_{0}\right)$.

Proof. Let $T$ be the Stone space of $\mathcal{F}$, whose corresponding space of real-valued continuous functions on $T, \mathcal{C}(T)$, can be linearly and isometrically identified with a closed vector subspace $\mathcal{S}$ of $\ell_{\infty}$ containing $c_{0}$. For every $n \in \mathbb{N}$, let us consider the continuous linear mapping $\sigma_{x^{n}}$ defined in (3.1), which will be simply denoted as $\sigma_{n}$, and let $\sigma_{n}^{0}$ be the restriction of $\sigma_{n}$ to $\mathcal{S}_{0}$, where $\mathcal{S}_{0}$ is the subspace of $\mathcal{S}$ composed by all sequences of finite range. Next,

$$
\lim _{n \rightarrow \infty} \omega A \sum_{k=1}^{\infty} b(k) x^{n}(k)
$$

exists for every $(b(k))_{k \in \mathbb{N}} \in \mathcal{S}_{0}$. Since $\mathcal{S}_{0}$ is barrelled (because it is linearly isometric to $\mathcal{C}_{0}(T)$ ), there exists $H>0$ such that $\left\|\sigma_{n}\right\|=\left\|\sigma_{n}^{0}\right\|<H$ for every $n \in \mathbb{N}$, and this, together with the fact that $\mathcal{S}_{0}$ is dense in $\mathcal{S}$, allows us to deduce that

$$
\lim _{n \rightarrow \infty} \omega A \sum_{k=1}^{\infty} a(k) x^{n}(k)
$$


exists for every $(a(k))_{k \in \mathbb{N}} \in \mathcal{S}$. Finally, $\mathcal{S}$ is an $\ell_{\infty}$-Grothendieck space (because $\mathcal{C}(T)$ has the Grothendieck property), so by applying Theorem 3.3 we deduce the existence of an element $x^{0} \in X\left(c_{0}\right)$ such that $\lim _{n \rightarrow \infty}\left\|x^{n}-x^{0}\right\|=0$ in $X\left(c_{0}\right)$.

To finish this section, we want to show an immediate consequence of the previous results and the fact that $X_{\omega}(\mathcal{S}, A)$ is complete (Theorem 2.2).

Corollary 3.6. Let $X$ be a real Banach space. Let $\left(x^{n}\right)_{n \in \mathbb{N}}$ be a sequence in $X_{\omega}(\mathcal{S}, A)$. Consider a closed vector subspace $\mathcal{S}$ of $\ell_{\infty}$ containing $c_{0}$ which is an $\ell_{\infty}$-Grothendieck space, and a regular matrix $A=\left(\alpha_{i j}\right)_{i, j \in \mathbb{N}}$. Then, $\left(x^{n}\right)_{n \in \mathbb{N}}$ is convergent in $X_{\omega}(\mathcal{S}, A)$ if and only if

$$
\lim _{n \rightarrow \infty} \omega A \sum_{k=1}^{\infty} a(k) x^{n}(k)
$$

exists for every $(a(k))_{k \in \mathbb{N}} \in \mathcal{S}$.

\section{REFERENCES}

1. A. Aizpuru and F.J. Pérez-Fernández, Spaces of $\mathcal{S}$-bounded multiplier convergent series, Acta Math. Hungar. 87 1-2 (2000) 135-146. MR1755883 (2001e:46029)

2. A. Aizpuru, A. Gutierrez-Dávila, and F.J. Pérez-Fernández, Boolean algebras and uniform convergence of series, J. Math. Anal. Appl. 284 (2003) 89-96. MR.1996119 (2004c:46020)

3. A. Aizpuru and C. Pérez-Eslava, Matrix methods in summability and weakly unconditionally Cauchy series, Preprint.

4. C. Bessaga and A. Pelczynski, On bases and unconditional convergence of series in Banach spaces, Studia Math. 17 (1958) 151-164. MR0115069 (22:5872)

5. J. Boos, Classical and Modern Methods in Summability, Oxford Mathematical Monographs, Oxford University Press, Oxford, 2000. MR 1817226 (2002b:40001)

6. Q. Bu and C. Wu, Unconditionally convergent series of operators on Banach spaces, J. Math. Anal. Appl. 207 (1997) 291-299. MR1438915 (98c:47001)

7. J. Diestel, Sequences and Series in Banach Spaces, Graduate Texts in Mathematics 92, New York, Springer-Verlag, 1984. MR737004 (85i:46020)

8. J. Diestel, H Jarchow, and A. Tonge, Absolutely Summing Operators, Cambridge Studies in Advanced Mathematics 43, Cambridge University Press, Cambridge, 1995. MR 1342297 (96i:46001)

9. R. Haydon, A nonreflexive Grothendieck space that does not contain $\ell_{\infty}$, Israel J. Math. 40 (1981) 65-73. MR636907 (83a:46028)

10. W. Schachermayer, On some classical measure-theoretic theorems for non-sigma-complete Boolean algebras, Dissertationes Math. (Rozprawy Mat.) 214 (1982) 33 pp. MR673286 (84d:28015)

11. C. Swartz, The Schur lemma for bounded multiplier convergent series, Math. Ann. 2633 (1983) 283-288. MR704294 (84h:46015)

Departamento de Matemáticas, Universidad de Cádiz, Puerto Real, Cádiz, 11510, SPAIN

E-mail address: antonio.aizpuru@uca.es

Department of Mathematical Sciences, Kent State University, Kent, Ohio, 44242

E-mail address: fgarcia@math.kent.edu

Departamento de Matemáticas, Universidad de Cádiz, Puerto Real, Cádiz, 11510, SPAIN

E-mail address: consuelo.perezeslava@alum.uca.es 